\title{
Peer reviewers 2012
}

The Editorial Team would like to thank the individuals listed below who generously gave their time and expertise in acting as peer reviewers for the Journal in 2012.

Abdala, Nadia
Abonyi, Sylvia
Afolabi, Ebenezer
Agampodi, Suneth
Aird, Ian
Ama, Njoku
Anderson, Claire
Andreadis, Natasha
Bacon, Lesley
Barber, Tristan
Barter, Janet
Beddard, Stuart
Belfield, Toni
Black, Kirsten
Bourke, Stephen
Brabin, Loretta
Briggs, Paula
Brin, Genevieve
Britton, Amanda
Brown, Sally
Bumpstead, Ewan
Caird, Lucy
Cameron, Sharon
Campbell, Martha
Church, Kathryn
Clarke, Jan
Coffey, Patricia
Connolly, Anne
Cook, Louise
Costa, Gilka
Aartion

\begin{tabular}{|c|c|}
\hline Cooper, Kevin & Hamoda, Haitham \\
\hline Crook, Angela & Hoggart, Lesley \\
\hline Crook, David & Hollingworth, Barbara \\
\hline Cruikshank, Maggie & Holt, Kelsey \\
\hline Culwell, Kelly & Homer, Caroline \\
\hline Devonald, Elizabeth & Husemeyer, Roy \\
\hline Dickson, Jane & Jans, Suze \\
\hline Dodge, Laura & Joffe, Carole \\
\hline Domoney, Claudine & Jones, Sian \\
\hline Dunn, Sheila & Joseph, Rosalind \\
\hline Edouard, Lindsay & Jungmann, Eva \\
\hline Eisenberg, Marla & Kalu, Emmanuel \\
\hline Elliott, Lawrie & Katusiime, Christine \\
\hline Elson, Janine & Kavanagh, Jayne \\
\hline Fielding, Shona & Kimambo, Ronald \\
\hline Flett, Gillian & Kingston, Margaret \\
\hline Forrest, Simon & Koren, Ainat \\
\hline Foster, Angel & Kuhl, Herbert \\
\hline French, Kathy & Lancastle, Deborah \\
\hline French, Rebecca & Latifnejed Rousari, Robab \\
\hline Frohwirth, Lori & Lee, Amanda \\
\hline Furedi, Anne & Lee, Ellie \\
\hline Gatiss, Sarah-Jane & Lim, Anita \\
\hline Gebbie, Ailsa & Lorimer, Karen \\
\hline Gibbon, Marion & Lundgren, Rebecca \\
\hline Glasier, Anna & Lyons, Deirdre \\
\hline Gollub, Erica & Macdonald, Sara \\
\hline Griffiths, Malcolm & MacGregor, Anne \\
\hline Guthrie, Kate & Mann, Susan \\
\hline Haider, Zara & Mansour, Diana \\
\hline
\end{tabular}

\begin{tabular}{|c|c|}
\hline Marions, Lena & Reid, Robert \\
\hline Massey, Louise & Romanowski, Barbara \\
\hline McCarthy, Michelle & Rowlands, Sam \\
\hline McEleny, Kevin & Salazar, Mariano \\
\hline Mehigan, Shelley & Sasieni, Peter \\
\hline Melvin, Louise & Sathiya Susuman, Appuni \\
\hline Mesher, David & Schunmann, Catherine \\
\hline Milsom, Ian & Serrant-Green, Laura \\
\hline Mittal, Monica & Sharma, Suresh \\
\hline Mollison, Jill & Shazly, Sherif \\
\hline Moore, Wendy & Shreffler, Karina \\
\hline Mortensen, Gitte & Shulman, Lee \\
\hline Mullin, Nicola & Snyder, Karrie \\
\hline Ngo, Anh & Soldan, Kate \\
\hline O’Brien, Paul & Stewart, Jane \\
\hline O’Sullivan, Lucia & Stone, Nicole \\
\hline Olowookere, Samuel & Stuart, Beth \\
\hline Oscarsson, Marie & Thomas, Sara \\
\hline Panay, Nick & Tristram, Amanda \\
\hline Parkes, Alison & Trussell, James \\
\hline Pauls, Alfred & Unger, Holger \\
\hline Peate, Michelle & Walker, Patrick \\
\hline Phillips, Karen & Waller, Jo \\
\hline Pillai, Mary & Warner, Pamela \\
\hline Pouget, Enrique & Waters, Laura \\
\hline Praet, Cecile & Webb, Anne \\
\hline Quinn, Gwendolyn & Weisberg, Edith \\
\hline Randall, Sarah & Weitz, Tracy \\
\hline Rank, Katherine & Wildt, Ludwig \\
\hline Reed, Craig & Zelin, Jill \\
\hline
\end{tabular}

